\title{
GENERALIZED MULTITIME EXPANSIONS FOR EQUATIONS WITH SLOWLY VARYING COEFFICIENTS
}

\author{
L.E. LEVINE and W.C. OBI \\ Department of Pure and Applied Mathematics \\ Stevens Institute of Technology \\ Hoboken, New Jersey 07030 \\ (Received April 18, 1983)
}

ABSTRACT. The successive terms in a uniformly valid multitime expansion of the solutions of constant coefficient differential equations containing a small parameter $\varepsilon$ may be obtained without resorting to secularity conditions if the time scales $t_{i}=\varepsilon^{i_{t}}(i=0,1, \ldots)$ are used. Similar results have been achieved in some cases for equations with variable coefficients by using nonlinear time scales generated from the equations themselves. This paper extends the latter approach to the general second order ordinary differential equation with slowly varying coefficients and examines the restrictions imposed by the method.

KEY WORDS AND PHRASES. Ordinary differential equations, asymptotic expansions, perturbations.

1983 MATHEMATICS SUBJECT CLASSIFICATION CODE. 34 E 05.

1. INTRODUCTION.

In multitime expansion techniques (also known as derivative-expansion methods), several time scales are used to obtain uniformly valid asymptotic expansions of the solutions of certain differential equations. Important a priori assumptions concerning the nature of these time scales and the successive terms of the expansions must be made in order to achieve acceptable results. Unfortunately, the justification for these assumptions is often based on intuitive insight and thus not readily transferable to other problems. Simple, formal techniques for choosing the time scales would be much more preferable. Results in this direction were given in [1] and [2]. It was shown that the succeeding terms in a multitime approximation to the solution of some problems could be obtained without recourse to secularity conditions. This simplified approach was based on results achieved by Reiss [3] for a lightly damped linear oscillator. The overall direction of this approach may be maintained in dealing with a fairly general class of second order ordinary differential equations. The method is a logical extension of the techniques developed in [1], [2], and [3] and is detailed in this article. Some of the results given here have been announced in 
abbreviated form in [4].

The general problem considered is

$\mathrm{L}_{\mathrm{sv}}[\mathrm{y}(\varepsilon t ; \varepsilon)] \equiv \mathrm{a}(\varepsilon t ; \varepsilon) \mathrm{y}^{\prime \prime}+\varepsilon b(\varepsilon t ; \varepsilon) \mathrm{y}^{\prime}+\mathrm{c}(\varepsilon t ; \varepsilon) \mathrm{y}=0$, (' means $\left.\frac{d}{d t}\right)$

with

$$
\mathrm{y}(0 ; \varepsilon)=\gamma \text {, and } \mathrm{y}^{\prime}(0 ; \varepsilon)=\delta \text {. }
$$

An approximation to the solution may be represented in the form

$$
y=\sum_{J=0}^{M} \varepsilon^{J} y^{J}\left(\tau ; t_{0}, \ldots, t_{N}\right)+R^{M}(t ; \varepsilon)
$$

The time scales $t_{0}, t_{1}, \ldots, t_{N}$ are linearly independent functions of $\tau=\varepsilon t$ and $\varepsilon$. If suitable restrictions are made on the functions $a, b$, and $c$, then these time scales may be determined from equation (1.1) using the same formal operations employed in [2]. The assumptions made in [2] regarding the approximating equation and the form of the zeroth order approximation are also made here. However, the general treatment given here does not impose a requirement for a uniformly valid expansion in an infinite domain, as was the case in [2]. Instead, generalized uniform asymptotic expansions (as developed in [4]) in some domains $D$ without turning points are obtained. The time scales used in these approximations are given by first order differential equations which can be integrated directly. Using the notation developed in [2], it follows that the time scales are determined by the following equations:

$$
\frac{\mathrm{dt}_{\mathrm{K}}}{\mathrm{dt}}=(\varepsilon / 2)^{\mathrm{K}} \mathrm{g}_{\mathrm{K}}(\tau ; \varepsilon), \mathrm{K}=0,1, \ldots
$$

The functions $g_{\mathrm{K}}(\tau ; \varepsilon)$ are determined recursively from

$$
\begin{aligned}
& \mathrm{g}_{\mathrm{K}} \equiv 0, \quad \mathrm{~K}<0 \\
& \mathrm{a} \mathrm{g}_{0}^{2}=\mathrm{c} \\
& 2 \text { a } g_{0} g_{1}=2\left(a g_{0}^{\prime}+b g_{0}\right) \\
& 2 \text { a } g_{0} g_{K}=-\sum \text { a } g_{i} g^{\prime}{ }_{\alpha}+2\left(a^{\prime} g_{K-1}^{\prime}+b g_{K-1}\right), K \geq 3 \text {, odd } \\
& i+j=k \\
& 1 \leq i, j \leq k-1 \\
& 2 \text { a } g_{0} g_{K}=\sum(-1)^{i+1} \text { a } g_{i} g_{j}-2\left(a^{\prime}{ }_{K-1}+b g_{K-1}\right) \text {, } \\
& i+j=k \\
& 1 \leq \mathrm{i}, \mathrm{j} \leq \mathrm{K}-1 \\
& K \geq 2 \text {, even. }
\end{aligned}
$$

In equations (1.5)-(1.9), the prime denotes differentiation with respect to $\tau=\varepsilon t$. In [2] the time scales determined by equations (1.4)-(1.5) could be expressed in closed form. Here, this is not necessarily the case. However, quadrature can be used to obtain numerical results from the expansions.

2. VALIDITY OF ASYMPTOTIC EXPANSIONS. The proof of the validity of the expansions described in $\S 1$ may be established 
by direct computations. The theorem given in [4] will be restated and proved here. THEOREM. Let the functions $a, b$, and $c$ be of constant sign and analytic in an interval $D$ such that $a$ and $c$ are never zero. If the time scales are determined from Equations (1.4) and (1.5), then for $N \geq 1,(1.3)$ is a $(M+1)$-term- $(N+1)$ - time generalized uniform asymptotic expansion of the solution of (1.1) and (1.2) in the interval D. The error $R^{M}(t ; \varepsilon)$ satisfies the inequalities

$$
\left|R^{N}(t ; \varepsilon)\right| \geq \begin{cases}\left|Y_{e}{ }^{M}\right| x|| U_{2}||+B /^{\varepsilon} & \text { if } M \text { is even } \\ \left|Y_{0}^{M}\right| x|| U_{2}||+\left.B\right|^{\varepsilon} & \text { if } M \text { is odd }\end{cases}
$$

with all the terms bounded in D.

(The definitions of the quantities appearing in (2.1) are given in the proof that follows.)

PROOF. The terms of the expansion (1.3) and the inequality (2.1) can be explicitly given because the time scales of (1.4) and (1.5) allow the steps detailed in [1] and [2] to be taken with similar results. The zeroth order approximation is given by

$$
\begin{aligned}
y^{0}\left(\tau ; t_{0}, \ldots, t_{N}\right) & =e^{-\left(t_{1}+t_{3}+\ldots+t_{H}\right)} \frac{1}{2}\left(\gamma-i\left(\delta / g_{0}(0)\right) e^{i\left(t_{0}+t_{2}+\ldots t_{2 n}\right)}\right. \\
& +\frac{1}{2}\left(\gamma+i\left(\delta / g_{0}(0)\right) e^{-i\left(t_{0}+t_{2}+\ldots+t_{2 n}\right)}\right.
\end{aligned}
$$

In general, the higher order terms of the expansion are of the form

$$
\begin{aligned}
y^{J}\left(\tau ; t_{0}, \ldots, t_{N}\right) & =\bar{e}^{\left(t_{1}+t_{3}+\ldots+t_{H}\right)} P_{J\left(t_{N}\right)} e^{i\left(t_{0}+t_{2}+\ldots+t_{2 n}\right)} \\
& +\bar{P}_{J}\left(t_{N}\right)-i\left(t_{0}+t_{2}+\ldots+t_{2 n}\right)
\end{aligned}
$$

where the polynominals $\mathrm{P}_{\mathrm{J}}\left(t_{\mathrm{N}}\right)$ may be generated recursively from a formula of the form

$$
\mathrm{P}_{\mathrm{J}+1}\left(\mathrm{t}_{\mathrm{N}}\right)=\mathrm{C}_{\mathrm{J}+1}+\mathrm{i} \int \mathrm{t}_{\mathrm{N}} \hat{\mathrm{P}}_{\mathrm{J}}\left({ }_{\sigma}\right)_{\mathrm{d} \sigma}
$$

The polynominals $P_{J}$ are complex valued $\left(\overline{\mathrm{P}}_{\mathrm{J}}\right.$ is the usual complex conjugate) and involve only the last and slowest time scale, $\mathrm{t}_{\mathrm{N}}$. The constants, $\mathrm{C}_{\mathrm{J}+1}$, are determined by the initial values $y^{\mathrm{J}+1}(0,0, \ldots, 0)$ and $\mathrm{y}_{\mathrm{t}_{0}}^{\mathrm{J}+1}(0,0, \ldots, 0)$ and it is
understood that

$$
\mathrm{P}_{\mathrm{J}} \equiv 0, \mathrm{C}_{\mathrm{J}} \equiv 0 \text { for } \mathrm{J}>0 \text {. }
$$

The subscripts $\mathrm{n}$ and $\mathrm{H}$ in (2.2) and (2.3) are defined by

$$
\mathrm{n}= \begin{cases}\mathrm{N} / 2 & \text { if } \mathrm{N} \text { is even } \\ (\mathrm{N}-1) / 2 \text { if } \mathrm{N} \text { is odd }\end{cases}
$$




$$
H= \begin{cases}N-1 & \text { if } N \text { is even } \\ N & \text { if } N \text { is odd }\end{cases}
$$

The recurrence relationships for obtaining the polynominals will be given in the appendix.

The error term satisfies the differential equation

$$
\mathrm{L}_{S V}\left[\mathrm{R}^{\mathrm{M}}\right]=\mathrm{r}^{\mathrm{M}}(\mathrm{t} ; \varepsilon)
$$

where the nonhomogeneous term $r^{M}=0\left(\varepsilon^{M+N+1}\right)$. Direct substitution using (2.2) and (2.3) enables us to express the initial conditions explicitly in terms of

$$
\begin{gathered}
R^{N}(0)=0 \\
\frac{d R^{M}}{d t}(0) \equiv\left\{\begin{array}{l}
Y_{e}^{M}=\varepsilon^{M+1}\left(\pi_{1} \gamma\right)+\varepsilon^{M+2}\left(\pi_{2} \delta\right)+\ldots \text { for } M \text { even } \\
Y_{0}^{M}=\varepsilon^{M+1}\left(\Lambda_{1} \delta\right)+\varepsilon^{M+2}\left(\Lambda_{2} \gamma\right)+\ldots \text { for } M \text { odd }
\end{array}\right.
\end{gathered}
$$

The quantities $\pi_{i}$ and $\Lambda_{i}, i=1,2, \ldots$, are constants independent of $\varepsilon$. Under the assumptions of the problem, a Green's function, $G^{*}$, may be obtained for the problem

$$
L[y]=y^{\prime \prime}+\frac{\varepsilon b(\varepsilon t ; \varepsilon)}{a(\varepsilon t ; \varepsilon)} y^{\prime}+\frac{c(\varepsilon t ; \varepsilon)}{a(\varepsilon t ; \varepsilon)} y=0
$$

in terms of its principal solutions $U_{1}$ and $U$, where

$$
\begin{aligned}
& \mathrm{U}_{1}(0)=1, \mathrm{U}_{1}^{\prime}(0)=0 \\
& \mathrm{U}_{2}(0)=0, \mathrm{U}_{2}^{\prime}(0)=1 .
\end{aligned}
$$

Expressing the solution of (2.8) and (2.9) formally in terms of $G^{*}$ and taking the bounds yields the inequality $(2.1)$ where

$$
\begin{aligned}
& B=\left\|\int_{0}^{t=\varepsilon t} G *(t, \sigma ; \varepsilon) \frac{r^{M}(\sigma ; \varepsilon)}{a(\sigma ; \varepsilon)} d \sigma\right\|, \\
& \|\cdot\|=\max _{t \in D} \mid: 1,
\end{aligned}
$$

and $|\cdot|$ denotes absolute value.

END OF PROOF.

3. CHARACTERISTICS OF THE EXPANSIONS.

The expansions obtained in the general case retain the characteristics of the earlier expansions of References [1] - [3]. The differential equation (1.1) may be satisfied to any degree of accuracy by merely increasing the number of time scales used. However, the approximation to the initial condition can be improved only by adding more terms to the expansion. Corollaries 2,3, and 4 of Reference [1] establishing the orders of magnitude of $R^{M}$ for different values of $N, M, \gamma$ and $\delta$ can be established for the general case. Thus, for certain choices of the initial conditions, accuracy may be gained by choosing expansions such that $M$ is either even or odd. Also, there is an isolated case in chich the zeroth order approximation 
may be used to obtain any degree of accuracy by increasing the number of time scales.

The expansions become secular in character as turning points are approached. This inadequacy of the expansions appears to be related to the assumed approximating equation. The extension of the technique to significantly different approximating equations has so far been unsuccessful. Such an extension may be necessary for handling turning points and other complications.

4. NUMERICAL APPLICATION.

The results of earlier works were reproduced by applying the general formulas of this paper. Then the expansions obtained were tested numerically against the exact solutions. Very good agreement was obtained as can be seen form Figures 1 and 2 . The figures show plots of the difference $|\Delta y|$, defomed by

$$
|\Delta y|=\mid y \text { exact }-\mathrm{y} \text { approx. } \mid
$$

plotted against time $t$. The computations were carried out with a PDP-10 computer.

Figure 1 evaluates the approximations of the solution to the problem

$$
(1+\varepsilon t) y^{\prime}+2 \varepsilon y^{\prime}+y=0,
$$

with

$$
y(0)=0 \text {, and } y^{\prime}(0)=1 \text {. }
$$

As reported in [2], the straightforward expansion is valid only in a small range of $t$ while the expansion obtained by our method is valid for $t \geq 0$. This is in evidence in the figure, where the straightforward expansion is 1abelled STF. The curves labelled I, II, and IV represent errors for a 1-term-2time expansion, a 1-term-3-time expansion, and a 3-term-3-time expansion, respectively. The expansions evaluated in Figure 1 are given in the Appendix.

Figure 2 illustrates the numerical results for the problem

$$
y^{\prime}+e^{-\varepsilon t} y=0
$$

with

$$
y(0)=0 \text {, and } y^{\prime}(0)=1
$$

Curve I gives the errors for a 1-term-2-time approximation; II gives the errors for a 1-term-4-time approximation while IV gives the results for a 3-term-4-time approximation. The approximations are given in the Appendix. Since equation (4.4) can be viewed as having a turning point at infinity, the expansions are not uniformly valid for all $t \geq 0$. But, they approximate the solutions very well for $t \geq \frac{1}{\varepsilon} \ln \frac{1}{\varepsilon}$, as can be seen from the figure. This is a great improvement over the straightforward expansion which is good in only a small interval. (The errors for the straightforward expansion fall out of the $t$ range in Figure 2.)

The order of magnitude estimates on which the theorem of $\S 2$ relied do not yield sufficient information for assessing numerical accuracy in cases like Equation (4.4). An assessment may be obtained by working directly with the error terms and the expansions, but it is tedious to get fine estimates in this manner. More realistic estimates of the interval in which the expansions yield numerically useful results may be obtained quickly by analysing the approximating equation. Since this equation has constant coefficients, the estimate may be based on the interval where the criterion for transforming $(4.4)$ to an equation with constant coefficients is most closely 
satisfied.

The expansions described in this paper are easily obtained, since the formulas have already been derived. They offer an effective means for obtaining numerical results; this would still be the case even if the time scales cannot be given in closed form. One of the most attractive features of the expansions is their ability to yield very high accuracy with a few terms.

5. APPENDIX

The term $\hat{P}_{J}$ under the integral sign of (2.4) is determined by the polynominals $P_{J}$ of the preceding terms of the expansion. The following symbols are required in order to write the expressions for $\mathrm{P}_{\mathrm{J}}$ and $\mathrm{P}_{\mathrm{J}}$ in a more compact form:

$$
\lambda(0, N)=2 a g_{0} g_{N}, \lambda(1, N)=a_{1} g_{N}, \ldots, \lambda(\nu, \mu)=2 a_{\nu} g_{\mu} / 2^{\nu+\mu-N}
$$

The first polynominal is

$$
P_{0}\left(T_{N}\right)=\frac{1}{2}\left[\gamma-i\left(\delta / g_{0}(0)\right)\right] .
$$

The second polynominal is given by

$$
\begin{aligned}
\lambda(0, N) P_{1}\left(t_{N}\right)=-(i / 2)\left[\lambda^{\circ}(1, N) / \lambda^{\circ}(0, N)\right] \lambda(0, N) & \\
+\left[\frac{1}{2}\left(\delta / g_{0}(0)\right) \lambda(0, N+1)+i\left(\frac{1}{2} \lambda(0, N+1)\right) \gamma\right] t & \text { if } N \text { is odd }
\end{aligned}
$$

or

$$
\begin{aligned}
& \lambda(0, \mathrm{~N}) \mathrm{P}_{1}=-(\mathrm{i} / 2)(\lambda)\left[\lambda^{\circ}(1, \mathrm{~N}) / \lambda^{\circ}(0, \mathrm{~N})\right] \lambda(0, \mathrm{~N}) \\
& +\left[-(1 / 2)(\gamma) \lambda(0, \mathrm{~N}+1)+(\mathrm{i} / \lambda)\left(\delta / \mathrm{g}_{0}(0)\right) \lambda(0, \mathrm{~N}+1] \mathrm{t}_{\mathrm{N}},\right. \\
& \text { if } \mathrm{N} \text { is even. }
\end{aligned}
$$

In equations (5.2) and (5.3), the superscript 0 in $\lambda^{\circ}(\nu, \mu)$ indicates that the function $\lambda(\nu, \mu)$ is evaluated at $\tau=0$. With $P_{0}$ and $P_{1}$ known, $P_{J}$ can be generated from the formulas

$$
\begin{aligned}
& \lambda(0, N) \hat{P}_{J} \equiv 1 / 2 \lambda(N, N) \dot{P}_{M+1-N^{-}} \sum_{S=0}^{n}\left[h(s, N) P_{J+1-(2 s+1)}-\lambda(0, N+2 s+1) x\right. \\
& P_{J+1-(2 s+1)]} \\
& +i\left\{\sum _ { r = 1 } ^ { n } \left[\lambda(N, 2 r) P^{\prime} J+1-2 r^{+}+\lambda(0, N+2 r) P_{J+1-2 r]}\right.\right.
\end{aligned}
$$

if $\mathrm{N}$ is odd

and

$$
\lambda(0, N) \hat{P}_{J} \equiv 1 / 2 \lambda(N, N) P_{J+1-N}^{\prime \prime} \sum_{s=0}^{n-1} h(s, N) P_{J+1-(2 s+1)}^{\prime}+\sum_{r=1}^{n} \lambda(0, N+2 r) P(J+1-2 r
$$




$$
+i\left\{\sum_{r=1}^{n} \lambda(N, 2 r) P^{\prime} J+1=2 r^{+} \sum_{s=0}^{n-1} \lambda(0, N+2 s+1) P J+1-(2 s+1)\right\}
$$

if $\mathrm{N}$ is even.

In equations (5.4) and (5.5), the prime (') denotes differentiation with respect to $t_{N}$. The function $h(\nu, \mu)$ is given by

$$
h(\nu, \mu)= \begin{cases}\lambda(1, \mu)-\left(\mathrm{ag}_{\mu}^{\prime}+\mathrm{bg}_{\mu},\right. & \text { if } \nu=0 \\ \lambda(2 \nu+1, \mu) & \text { if } \nu>0\end{cases}
$$

The time scales generated from (4.2) are

$$
t_{0}=(2 / \varepsilon)(\sqrt{1+\tau}-1), t_{1}=(3 / 4) \ln (1+t)
$$

and

$$
t_{2}=(3 \varepsilon / 16)[1 / \sqrt{1+\tau}-1], t_{3}=\left(3 \varepsilon^{2} / 64\right)[1-1 /(1+\tau)] .
$$

The expansions tested in Figure 1 are as follows:

$$
\begin{aligned}
y & =y^{0}+0(\varepsilon)=e^{-t_{1}} \sin t_{0}+0(\varepsilon) \\
y & =y^{0}+0\left(\varepsilon^{2}\right)=e^{-t} 1_{1} \sin \left(t_{0}+t_{2}\right)+0\left(\varepsilon^{2}\right) \\
y & =y^{0}+\varepsilon y^{1}+\varepsilon^{2} y^{2}+0\left(\varepsilon^{4}\right) \\
& =e^{-t_{1}} \sin \left(t_{0}+t_{2}\right)+\varepsilon\left[t_{2} 2(1+\tau)^{1 / 2}\right] e^{-t} \sin \left(t_{0}+t_{2}\right) \\
& +\varepsilon^{2}\left[\left(t_{2} / 8(1+t)\right) e^{-t} 1 \cos \left(t_{0}+t_{1}\right)\right. \\
& \left.+\left(3 / 32+t_{2}^{2} / 2(1+t)\right) e^{-t_{1}} \sin \left(t_{0}+t_{2}\right)\right]+0\left(\varepsilon^{4}\right)
\end{aligned}
$$

The straightforward expansion for the solution of equations (4.2) and (4.3) is

$$
y=\sin t=\varepsilon / 4\left(3 t \sin t+t^{2} \cos t\right)+0\left(\varepsilon^{2}\right) \text {. }
$$

The time scales generated from Equation (4.4) are

$$
\begin{aligned}
& t_{0}=2 / \varepsilon\left[1-e^{-\tau / 2}\right], t_{1}=-{ }^{\tau} / 4, \\
& t_{2}=\varepsilon / 10\left[e^{\tau / 2}-1\right], \text { and } t_{3}=\varepsilon^{2 /} 64\left[e^{\tau}-1\right] .
\end{aligned}
$$

The expansions evalueated in Figure 2 are:

$$
\begin{aligned}
& y=y^{0}+o(\varepsilon)=e^{-t_{1}} \operatorname{sint}_{0}+o(\varepsilon) \\
& y=y^{0}+0\left(\varepsilon^{2}\right)=\bar{e}^{-\left(t_{1}+t_{3}\right)} \sin \left(t_{0}+t_{2}\right)+0\left(\varepsilon^{2}\right) \\
& \mathrm{y}=\mathrm{y}^{0}+\varepsilon \mathrm{y}^{1}+\varepsilon^{2} \mathrm{y}^{2}+0\left(\varepsilon^{4}\right) \\
& =\bar{e}^{-\left(t_{1}+t_{3}\right) \sin \left(t_{0}+t_{2}\right)+\varepsilon\left[-25 / 32 t_{3} e^{\tau} / 2\right] e^{-\left(t_{1}+t_{3}\right)} \cos \left(t_{0}+t_{2}\right)} \\
& +\varepsilon^{2}\left[-1 / 32+79 / 128 t_{3} e^{\tau}-8(25 / 128)^{2} t_{3}{ }^{2} e^{\tau}\right] \\
& x \bar{e}^{-\left(t_{1}+t_{3}\right)} \sin \left(t_{0}+t_{2}\right)+0\left(\varepsilon^{4}\right)
\end{aligned}
$$

\section{REFERENCES}

1. OBI, W.C., "A Class of Generalized Uniform Asymptotic Expansions", Internat. J. Math and Math. Sci., $\underline{1}$ (1978) 161-175.

2. LEVINE, L.E. and OBI, W.C., "Multivariable Expansion Using Time Scales Generated From Differential Equations", SIAM J. APPL. MATH., 33, No. 4. Dec. (1977). 
3. REISS, E.L., "On Multivariable Asymptotic Expansions", SIAM Rev. 13 (1971) 189-196.

4. LEVINE, L.E. and OBI, W.C., "Multivariable Expansion of Solutions of Linear Equations with Slowly Varying Coefficients", Bulletin American Math. Soc., 82, (1976). 


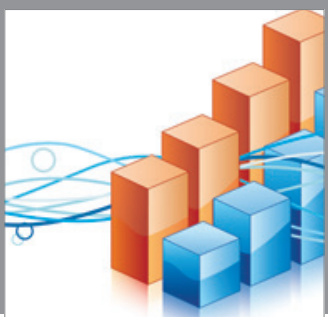

Advances in

Operations Research

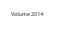

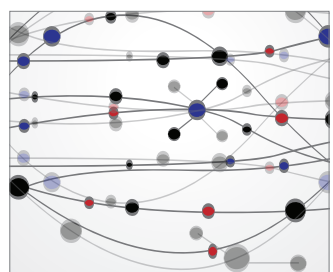

\section{The Scientific} World Journal
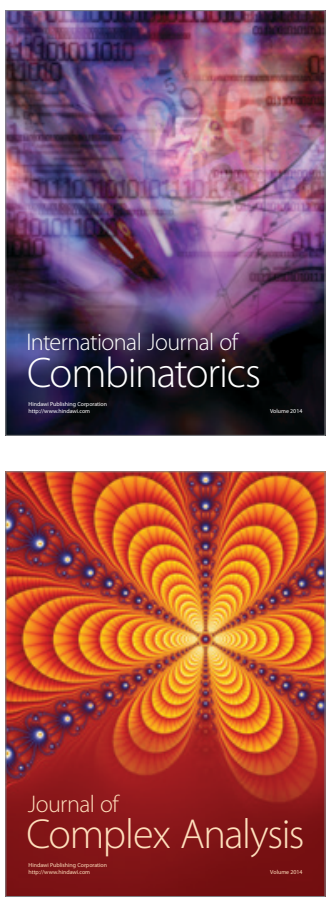

International Journal of

Mathematics and

Mathematical

Sciences
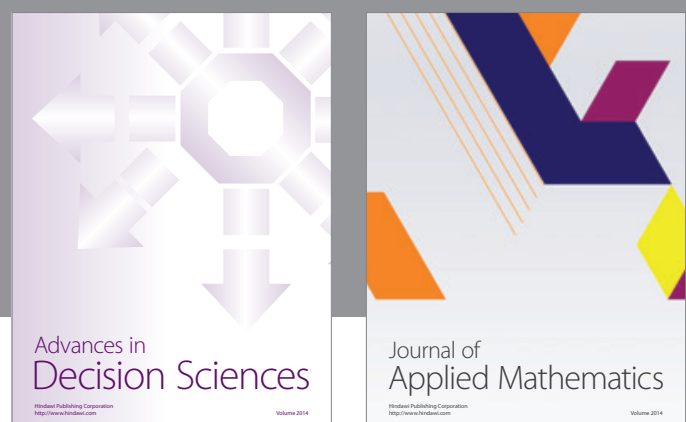

Journal of

Applied Mathematics
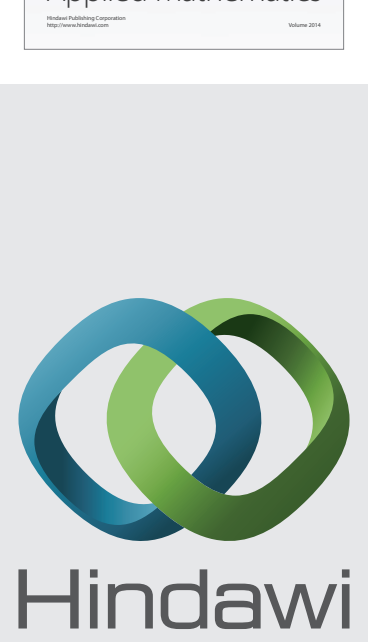

Submit your manuscripts at http://www.hindawi.com
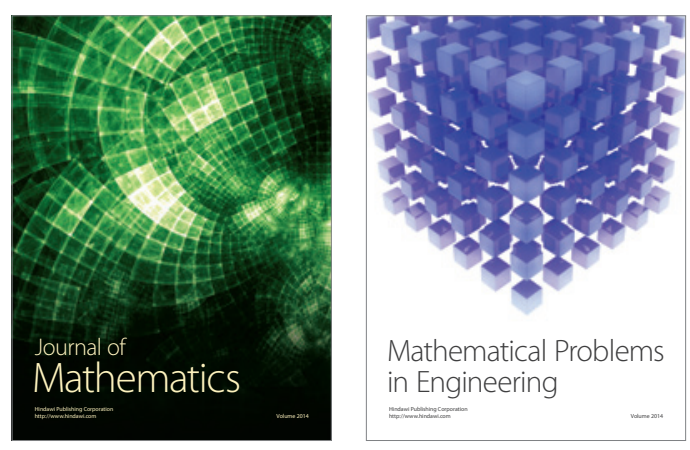

Mathematical Problems in Engineering
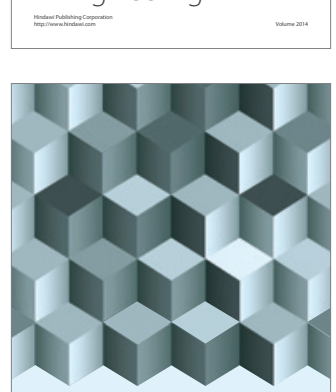

Journal of

Function Spaces
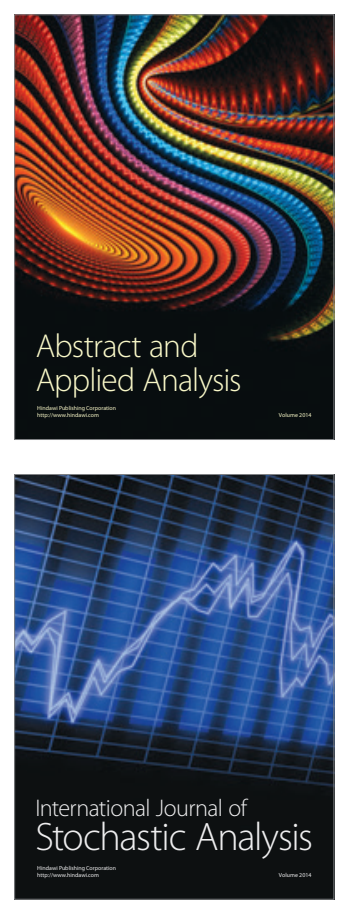

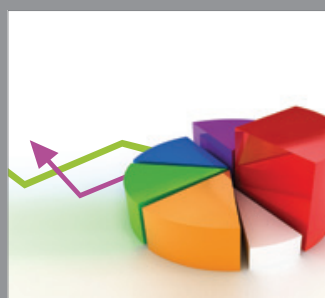

ournal of

Probability and Statistics

Promensencen
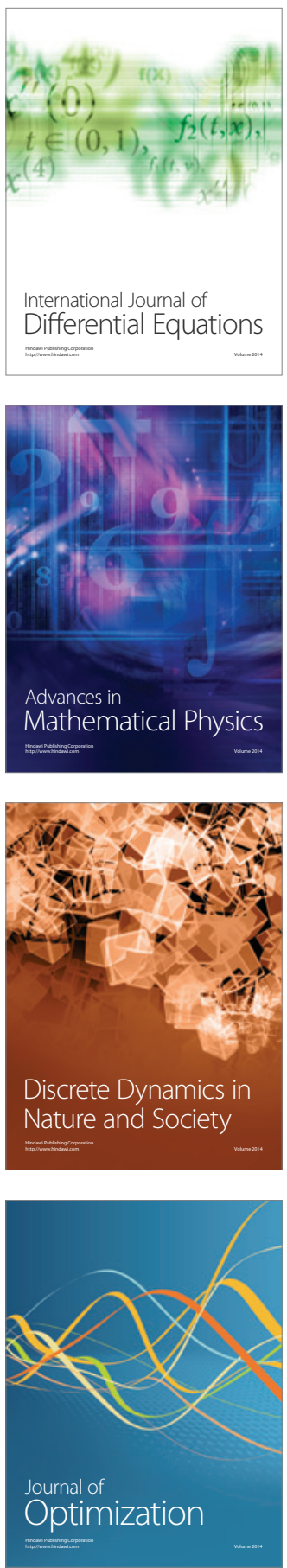